\title{
Downregulation of BCL11A by siRNA induces apoptosis in B lymphoma cell lines
}

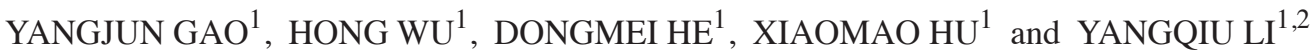 \\ ${ }^{1}$ Institute of Hematology, Medical College; ${ }^{2}$ Key Laboratory for Regenerative Medicine of \\ Ministry of Education, Jinan University, Guangzhou, Guangdong 510632, P.R. China
}

Received June 26, 2012; Accepted August 28, 2012

DOI: $10.3892 /$ br.2012.9

\begin{abstract}
The B-cell chronic lymphocytic leukemia (CLL)/lymphoma 11A gene (BCL11A) encodes a krüppel-like zinc finger protein, which is important in thymopoiesis and has been associated with hematopoietic malignancies. In this study, we investigated whether the downregulation of BCL11A mRNA by small interference RNA (siRNA) was capable of inducing apoptosis, and tested the effect of BCL11A siRNA combined with BCL2 siRNA in B lymphoma cell lines (SUDHL6, EB1). BCL11A siRNA was transfected into SUDHL6, EB1 cells with HiPerfect transfection reagents. After transient transfection with BCL11A siRNA, the expression levels of BCL11A mRNA and protein were assayed by quantitative reverse transcription polymerase chain reaction (qRT-PCR) and western blot analysis. The cell proliferation was determined by a cell counting kit-8 (CCK8) assay. Apoptosis was determined by morphological observation and flow cytometric analysis. The results showed that the expression levels of BCL11A mRNA and protein from SUDHL6, EB1 cells transfected with BCL11A siRNA decreased, compared with either the scrambled negative control siRNA group or untransfected cells group $(\mathrm{P}<0.05)$. Viability of cells transfected with BCL11A siRNA was less compared to cells transfected with control siRNA and untransfected SUDHL6, EB1 cells, respectively $(\mathrm{P}<0.05)$. BCL11A siRNA induced apoptosis in both SUDHL6 and EB1 cells. BCL11A siRNA combined with BCL2 siRNA significantly inhibited cell growth. Apoptotic rates of SUDHL6, EB1 cells treated with BCL11A siRNA combined with BCL2 siRNA significantly increased $(\mathrm{P}<0.05)$, compared with either the scrambled control (Sc) siRNA and BCL2 siRNA combination or BCL2 or BCL11A siRNA-treated cells alone. Findings of this study suggest the downregulation of BCL11A mRNA by siRNA was able to induce apoptosis.
\end{abstract}

Correspondence to: Dr Dongmei He, Institute of Hematology, Medical College, Jinan University, No. 601 West Huangpudadao, Guangzhou, Guangdong 510632, P.R. China

E-mail: thedm@jnu.edu.cn

Key words: B-cell chronic lymphocytic leukemia/lymphoma 11A, siRNA small interfering RNA, SUDHL6 cells, EB1 cells, BCL2, apoptosis
Moreover, BCL11A siRNA combined with BCL2 siRNA increased apoptosis in SUDHL6, EB1 cells. Thus, suppression of BCL11A expression may be a useful approach in the treatment of B lymphoma.

\section{Introduction}

The B-cell chronic lymphocytic leukemia (CLL)/lymphoma 11 (BCL11) gene family members, including the BCL11A and BCL11B genes, encode krüppel-like $\mathrm{C}_{2} \mathrm{H}_{2}$ zinc finger proteins, which initially have been identified as transcriptional repressors (1-3). The BCL11A gene was initially found in mice as an ecotropic retrovirus integration site (Evi9), also known as chicken ovalbumin upstream promoter transcription factor-interacting protein 1 (CTIP1) (1). BCL11A was previously shown to be associated with lymphocyte proliferation and differentiation $(2,4)$. Abnormalities involving BCL11A have been detected in a variety of B-cell malignancies and certain subsets of acute myeloid leukemia in humans (3,5-6). We and others reported high levels of BCL11A mRNA expression in the majority of B-CLL, B-lymphoma and B-cell leukemia/lymphoma cell lines (3,7-10). However, the precise function of this transcription factor in B-cell malignancies still remains poorly characterized. Little is known whether the BCL11A gene is associated with cell apoptosis. Double-stranded RNA-dependent post-transcriptional gene silencing, also known as RNA interference (RNAi), is a phenomenon where double-stranded small interfering RNA (siRNA) complexes are able to target specific genes. In the present study, we firstly report that the suppression of BCL11A by RNAi is capable of inducing apoptosis in the diffuse large B-cell lymphoma (SUDHL6) and Burkitt's lymphoma (EB1) cell lines.

BCL2, a member of the BCL2 family, plays an important role in programmed cell death/apotosis (11). It has been shown that siRNA targeting against BCL2 mRNA was able to effectively induce apoptosis of lymphoma and leukemia cells (11-13). However, whether BCL11A siRNA combined with BCL2 siRNA enhanced apoptosis of lymphoma SUDHL6, EB1 cells remains uncertain. Therefore, we also investigated whether BCL11A siRNA combined with BCL2 siRNA was able to enhance cell apoptosis. The findings indicated that the downregulation of BCL11A serves as a new therapeutic option in B-cell malignancies. 


\section{Materials and methods}

Reagents. BCL11A-specific (BCL11A-585,2292) siRNAs, BCL2-specific (BCL2-195) siRNA and the corresponding non-silencing scrambled negative control (Sc) siRNA were designed and synthesized by Shanghai GenePharma Co., Ltd. (Shanghai, China). RPMI-1640 and newborn calf serum were purchased from Gibco (Carlsbad, CA, USA).

Cell culture and transfection with siRNA. The SUDHL6, EB1 cell lines were kindly provided by Professor Ailin Guo from the Department of Plant Pathology (Cornell University, Ithaca, NY, USA). The cells were cultured in RPMI-1640 supplemented with $10 \%$ heat-inactivated fetal calf serum at $37^{\circ} \mathrm{C}$ in a humidified incubator at $5 \% \mathrm{CO}_{2}$. SUDHL6, EB1 cells in the exponential phase of growth were grown for $24 \mathrm{~h}$, and transfected with BCL11A siRNA (si585, si2292) using HiPerfect (Qiagen, Valencia, CA, USA), following the manufacturer's protocols. Mock control cells (mock) and sc siRNA were transfected with HiPerfect agents. The total concentration of siRNA applied in each case was kept constant at 100 nM. For treatments with combined BCL11A siRNA and BCL2 siRNA, the ratio of BCL11A siRNA to BCL2 siRNA was kept at 1:1. At 48 and $72 \mathrm{~h}$ of transfection, silencing of BCL11A gene was examined.

Assay of BCL11A mRNA by $q R T-P C R$. Total RNA was isolated by TRIzol ${ }^{\circledR}$ total RNA isolation system (Invitrogen Life Technologies, Carlsbad, CA, USA) and reverse transcribed into the first-strand complementary DNA (cDNA) using random hexamer primers and the reverse transcriptase Superscript $\mathrm{II}^{\circledR}$ kit (Invitrogen Life Technologies), according to the manufacturer's instructions. RNA purity and concentration was measured with a spectrophotometer. The $2^{-\Delta \mathrm{Ct}} \times 100 \%$ method $[\Delta \mathrm{Ct}=\mathrm{CT}(\mathrm{BCL} 11 \mathrm{~A})-\mathrm{CT}(\mathrm{GAPDH})]$ was used to analyze the relative changes in gene expression from qRT-PCR experiments with SYBR-Green I $(14,15)$. The primers were synthesized (Shanghai Genechem Biotechnology Co., Ltd., China) as previously described $(16,17)$. The primer sequences used in the PCR reactions were: BCL11A sense: 5'-AACCCCA GCACTTAAGCAAA-3' and antisense: 5'-GGAGGTCATGA TCCCCTTCT-3'; GAPDH sense: 5'-ACCCAGAAGACTGTG GATGG-3' and antisense: 5'-TTCAGCTCAGGGATGAC CTT-3'. The total reaction volume was $20 \mu \mathrm{l}$. Reaction conditions were initiated with the enzyme activation at $95^{\circ} \mathrm{C}$ for $10 \mathrm{~min}$, followed by 40 cycles at $95^{\circ} \mathrm{C}$ for $15 \mathrm{sec}, 60^{\circ} \mathrm{C}$ for $30 \mathrm{sec}$ and $80^{\circ} \mathrm{C}$ for $5 \mathrm{sec}$. At the end of each run a melting curve was performed starting at a temperature of $65^{\circ} \mathrm{C}$ and rising up to $95^{\circ} \mathrm{C}$ with an increase of $1^{\circ} \mathrm{C}$ per $2 \mathrm{sec}$ to verify primer specificities. Each run was completed with a melting curve analysis to confirm the specificity of the amplification and the absence of primer dimmers. The qRT-PCR was repeated in at least three separate experiments.

Western blot analysis. Cells were washed with phosphatebuffered saline (PBS) (10 mM, pH 7.4), incubated in $200 \mathrm{ml}$ of cell lysis buffer $(50 \mathrm{mmol} / \mathrm{l}$ Tris- $\mathrm{HCl}, 5 \mathrm{mmol} / \mathrm{l}$ EDTA, $25 \%$ sucrose, $0.6 \mathrm{ml}$ of $100 \mathrm{mg} / \mathrm{ml}$ phenylmethylsulphonyl fluoride, $2.4 \mathrm{ml}$ 2-mexca-ptoethanol) on ice for $30 \mathrm{~min}$, and centrifuged at $13,000 \mathrm{x}$ g for $45 \mathrm{~min}$ at $4^{\circ} \mathrm{C}$. The protein of cell lysate was determined by a Bio-Rad Protein assay according to the manufacturer's instructions (Bio-Rad, Hercules, CA, USA). Whole cell extracts equivalent to $100 \mu \mathrm{g}$ of total protein were separated by $8 \%$ sodium dodecyl sulfate (SDS) polyacrylamide gel electrophoresis and electrotransferred to nitrocellulose membranes (Gibco-BRL, Carlsbad, CA, USA). The blot was placed in blocking buffer (10\% non-fat dry milk, 1\% Tween-20; in $20 \mathrm{mM}$ Tris-buffered saline, $\mathrm{pH}$ 7.5) for $1 \mathrm{~h}$ at room temperature and incubated with appropriate anti-human primary antibody (Abcam, Cambridge, MA, USA; mouse anti-human BCL11A/IgG, 1:1,000; mouse anti-human GAPDH/IgG, 1:2000) overnight at $4^{\circ} \mathrm{C}$. Blots were incubated with anti-mouse horseradish peroxidase-conjugated secondary antibody $(1: 3,000)$ for $1 \mathrm{~h}$ and detected by chemiluminescence using ECL Hyperfilm.

Assay of cell viability. For the quantitative determination of cellular proliferative activity, the cell counting kit-8 (CCK8; Dojindo, Tokyo, Japan) assay was carried out following transfection of SUDHL6 and EB1 cells with BCL11A siRNA alone or in combination with BCL2 siRNA at 24, 48 and $72 \mathrm{~h}$. The cells were washed, counted and seeded at a density of $4 \times 10^{5} / \mathrm{ml}$ cells per well in 96-well plates. Six hours later, BCL11A siRNA alone or in combination with BCL2 siRNA was added to the cells. At 24, 48 and $72 \mathrm{~h}$ after transfection, CCK8 solution was added $4 \mathrm{~h}$ before the end of incubation. Cell viability was measured with a spectrophotometer at an absorbance of $450 \mathrm{~nm}$. The inhibition rates of cell growth were calculated according to the formula: inhibition rate $(\%)=(1-$ mean absorbance of treatment group/mean absorbance of untreatment group) $\mathrm{x} 100 \%$.

Cell apoptosis assay. The transfected SUDHL6, EB1 cells were harvested after treatment. Morphology was determined with Hoechst 33258 (Invitrogen) following incubation for $72 \mathrm{~h}$. Cells were washed with PBS three times and then stained with $10 \mu \mathrm{l}$ Hoechst 33342 nuclear dye for $10 \mathrm{~min}$ at $37^{\circ} \mathrm{C}$. Cells were again washed three times with PBS and images were obtained by fluorescence microscopy (Leica, Wetzlar, Germany). Apoptosis detection kit (Annexin V BioVision, Palo Alto, CA, USA) was performed as per the manufacturer's instructions. Briefly, cells were centrifuged, washed with cold PBS, and resuspended in $500 \mu 1$ binding buffer. Fluorescein isothiocyanate-conjugated Annexin V $(10 \mu \mathrm{l})$ and propidium iodide (PI) $(10 \mu \mathrm{l})$ were added to each sample, and the mixture was incubated at $4^{\circ} \mathrm{C}$ in the dark for $5 \mathrm{~min}$. The cells were immediately subjected to fluorescence-activated cell sorting (FACS) analysis (BD FACSCalibur; BD Biosciences, San Jose, CA, USA). The percentage of the early and late apoptotic cells in each group was determined.

Statistical analysis. Data were shown as the mean \pm standard deviation (SD). Statistical comparisons were made by analysis of variance (ANOVA). Differences were deemed significant at $\alpha=0.05$. Statistical analysis was carried out using SPSS 13.0.

\section{Results}

BCL11A siRNAs suppressed BCL11A expression at the $m R N A$ and protein levels in SUDHL6 and EB1 cells. To determine the 
Table I. Effect of BCL11A siRNA on BCL11A mRNA expression levels by qRT-PCR at $48 \mathrm{~h}$ after transfection in SUDHL6 and EB 1 cells $(\%$, mean $\pm \mathrm{SD}, \mathrm{n}=3)$.

\begin{tabular}{lcccr}
\hline Cell lines & si585 & si2292 & Sc & Mock \\
\hline SUDHL6 & $36.91 \pm 2.63^{\mathrm{a}}$ & $37.73 \pm 1.97^{\mathrm{a}}$ & $62.11 \pm 2.57$ & $63.43 \pm 2.69$ \\
EB1 & $32.09 \pm 2.08^{\mathrm{a}}$ & $38.69 \pm 3.62^{\mathrm{a}}$ & $51.46 \pm 3.37$ & $53.86 \pm 1.93$ \\
\hline
\end{tabular}

${ }^{\mathrm{a}} \mathrm{P}<0.05$; SD, standard deviation; qRT-PCR, quantitative reverse transcription polymerase chain reaction; Sc, scrambled control.

Table II. Inhibition rates of SUDHL6 and EB1 cell growth by CCK8 assay after transfection with BCL11A siRNA $(\%, \pm \mathrm{SD}, \mathrm{n}=3)$.

\begin{tabular}{|c|c|c|c|c|}
\hline Cell lines & si585 & si2292 & Mock & $\mathrm{Sc}$ \\
\hline \multicolumn{5}{|c|}{ SUDHL6 (h) } \\
\hline 24 & $9.66 \pm 1.65^{\mathrm{a}}$ & $12.96 \pm 1.16^{\mathrm{a}}$ & $3.39 \pm 0.79$ & $4.43 \pm 0.85$ \\
\hline 48 & $39.70 \pm 2.95^{\mathrm{a}}$ & $36.19 \pm 1.34^{\mathrm{a}}$ & $3.23 \pm 1.53$ & $9.63 \pm 2.84$ \\
\hline 72 & $44.90 \pm 2.47^{\mathrm{a}}$ & $42.27 \pm 4.10^{*}$ & $3.23 \pm 0.86$ & $9.39 \pm 2.41$ \\
\hline \multicolumn{5}{|l|}{ EB1 (h) } \\
\hline 24 & $35.54 \pm 2.17^{\mathrm{a}}$ & $15.21 \pm 2.09^{\mathrm{a}}$ & $5.91 \pm 0.27$ & $3.52 \pm 1.17$ \\
\hline 48 & $37.91 \pm 3.34^{\mathrm{a}}$ & $33.48 \pm 2.75^{\mathrm{a}}$ & $3.85 \pm 0.69$ & $8.01 \pm 2.60$ \\
\hline 72 & $44.38 \pm 6.21^{\mathrm{a}}$ & $38.42 \pm 3.67^{\mathrm{a}}$ & $3.54 \pm 1.21$ & $2.37 \pm 0.87$ \\
\hline
\end{tabular}

${ }^{\mathrm{a}} \mathrm{P}<0.05$; CCK8, cell counting kit- 8 ; SD, standard deviation; Sc, scrambled control.

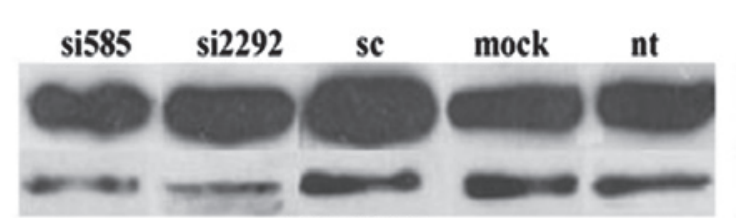

SUDHL6

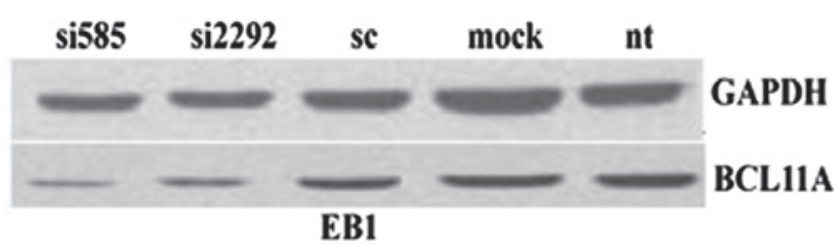

Figure 1. Effect of BCL11A siRNA on BCL11A protein expression levels in SUDHL6 and EB1 cells. BCL11A protein levels were determined using western blot analysis at $72 \mathrm{~h}$ after transfection with BCL11 siRNA585 (si585) or BCL11A siRNA2292 (si2292) is shown. Non-silencing scrambled siRNA (Sc)-treated, mock-transfected (mock) and non-treated (nt) cells were used as controls. GAPDH served as a loading control.

efficiency of BCL11A inhibition following siRNA treatment, BCL11A expression was analyzed at the mRNA and protein level. The qRT-PCR data shown include at least three independent experiments. According to the relative qRT-PCR formula: $2^{-\Delta \mathrm{Ct}} \times 100 \%(14,15)$ the relative expression levels of BCL11A mRNA in SUDHL6 and EB1 cells treated with BCL11A siRNA (siRNA585 and 2292) were significantly lower than cells treated with the control siRNA $(\mathrm{P}<0.05)$, at $48 \mathrm{~h}$ after transient transfection (Table I). By contrast, the control siRNA had no effect on the expression of BCL11A mRNA.

BCL11A protein levels were assayed by western blot analysis. Western blot analysis confirmed the results of the qRT-PCR analysis (Fig. 1). Expression of BCL11A protein significantly decreased in the two cell lines treated with BCL11A siRNA as compared with either the negative control siRNA group or untreated cells group. No difference in BCL11A levels among the control siRNA group, mock-transfected-treated cells and untransfected cells was found. The two BCL11A siRNAs were equally effective at reducing BCL11A mRNA and protein. No statistically significant difference was detected between the two cancer cell lines.

The effects of BCL11A siRNA on cell proliferation in SUDHL6 and $E B 1$ cells. For the quantitative determination of proliferative activity, a CCK8 assay was carried out. A summary of experiments measuring cell proliferation at 24, 48 and $72 \mathrm{~h}$ is shown in Table II. The growth of SUDHL6 and EB1 cells was inhibited in a time-dependent manner at a concentration of $100 \mathrm{nM}$ siRNA (siRNA585 and 2292) 24, 48 and $72 \mathrm{~h}$ after transfection. Of note, a difference was detected in cell viability between siRNA585 and 2292 at 24 h in EB1 cells. However, no statistically significant difference was found between siRNA585 and 2292 at 48 and $72 \mathrm{~h}$, nor between the two cell lines. 
Table III. Rate of apoptosis by Annexin V analysis at $72 \mathrm{~h}$ after transfection with BCL11A siRNA in SUDHL6 and EB1 cells $(\%$, mean $\pm \mathrm{SD}, \mathrm{n}=3)$.

\begin{tabular}{lccccr}
\hline Cell lines & si585 & si2292 & Sc & Mock & Cells \\
\hline SUDHL6 & $32.00 \pm 3.15^{\mathrm{a}}$ & $26.53 \pm 2.94^{\mathrm{a}}$ & $5.12 \pm 3.18$ & $2.90 \pm 2.37$ & $2.11 \pm 1.39$ \\
EB1 & $29.43 \pm 2.38^{\mathrm{a}}$ & $23.90 \pm 2.76^{\mathrm{a}}$ & $4.66 \pm 2.12$ & $3.24 \pm 1.17$ & $1.76 \pm 0.48$ \\
\hline
\end{tabular}

${ }^{\mathrm{a}} \mathrm{P}<0.05 ; \mathrm{SD}$, standard deviation; Sc, scrambled control.
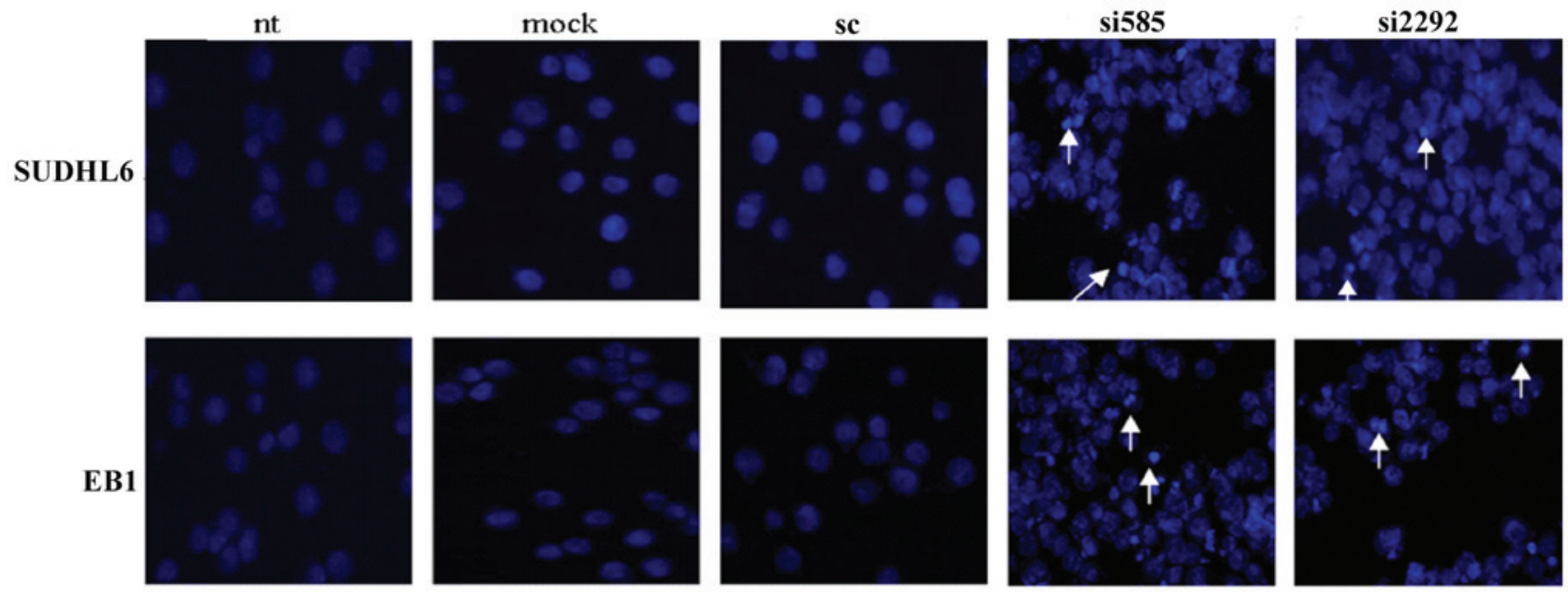

Figure 2. The morphology effect of BCL11A siRNA on apoptosis of SUDHL6 and EB1 cells. Nuclear morphology of cells stained with Hoechst 33258 was analyzed by fluorescence microscopy (magnification, x200) at $72 \mathrm{~h}$ after transfection with BCL11A siRNA (si585, si2292). Non-silencing scrambled siRNA (Sc), mock-transfected (mock) and untreated (nt) cells were used as controls. Data are representative microscopic images.

Effects of BCL11A siRNA on cell apoptosis in SUDHL6 and EBI cells. Two methods were used to assess apoptosis: i) Hoechst staining and ii) Annexin V-FITC/PI double staining by FACS analysis. SUDHL6, EB1 cells exhibited characteristic of apoptosis. As shown in Fig. 2, in the control groups, the cells demonstrated normal nuclear morphology, while at $72 \mathrm{~h}$ after transfection with BCL11A siRNA in SUDHL6, EB1 cells, a significant nuclear condensation and fragmentation were observed. Annexin V-FITC and PI double staining with FACS were employed to detect the effect of BCL11A siRNA on cell apoptosis. The percentage of Annexin V-FITC/ PI-stained cells for early and late apoptotic cells increased, reaching 32.00 and $29.43 \%$ for siRNA585 and 26.53 and $23.90 \%$ for siRNA2292 at $72 \mathrm{~h}$ in the SUDHL6 and EB1 cell lines, respectively (Table III). Control siRNA-treated, -untreated or mock-transfected cells showed $\sim 1-5 \%$ apoptotic cells. No statistically significant difference was detected in cell apoptosis between siRNA585 and 2292, nor between the two cell lines.

Effects of BCL11A siRNA in combination with BCL2 siRNA on cell growth and apoptosis in SUDHL6 and EBI cells. To investigate the effects of BCL11A siRNA combined with BCL2 siRNA on SUDHL6 and EB1 cells, a CCK8 assay was applied. A summary of experiments measuring cell proliferation at 24, 48 and $72 \mathrm{~h}$ is shown in Table IV. Compared to treatments with either BCL11A siRNA or BCL2 siRNA alone, cell viability was effectively reduced by combining BCL11A siRNA and BCL2 siRNA in a time-dependent manner. No difference in cell viability was detected between siRNA585 combined with BCL2 siRNA and siRNA2292 combined with BCL2 siRNA. A large increase was found in AnnexinV-FITC/PI double-positive cells at $72 \mathrm{~h}$ after the combined treatment with BCL11A siRNA and BCL2 siRNA in SUDHL6, EB1 cells (Table V). The difference in apoptotic rates was significant, compared to BCL11A siRNA or BCL2 siRNA alone, respectively $(\mathrm{P}<0.05)$. No significant difference in apoptosis occurred between those cells treated with siRNA585 combined with BCL2 siRNA and siRNA2292 combined with BCL2 siRNA ( $>0.05)$.

\section{Discussion}

The BCL11A gene encodes a $\mathrm{C}_{2} \mathrm{H}_{2}$ zinc finger protein, which is a krüppel transcription factor and is closely associated with B cell proliferation and differentiation $(2,4)$. Alternative splicing within the BCL11A locus leads to the creation of four major protein isoforms: extra long (XL), long (L), short (S), and extra short (XS) $(3,7)$. The BCL11A-XL mRNA is the largest and most abundant transcript (3). Our previous results showed the expression level of BCL11A in the B-cell acute lymphocyte leukemia patients and B lymphoma cell lines (including SUDHL6 and EB1) to be increased significantly, when compared to the T-cell acute lymphocyte leukemia and 
Table IV. Inhibition rates of SUDHL6 and EB1 cell growth by CCK8 assay following transfection with BCL11A siRNA in combination with BCL2 siRNA $(\%, \pm \mathrm{SD}, \mathrm{n}=3)$.

\begin{tabular}{lrrrrr}
\hline Cell lines & \multicolumn{1}{c}{ si585 } & si2292 & siBCL2 & si585+siBCL2 & si2292+siBCL2 \\
\hline SUDHL6 (h) & & & & & \\
24 & $9.66 \pm 1.65$ & $12.96 \pm 1.16$ & $14.2 \pm 2.25$ & $53.42 \pm 4.87^{\mathrm{a}}$ & $48.10 \pm 3.90^{\mathrm{a}}$ \\
48 & $39.70 \pm 2.95$ & $36.19 \pm 1.34$ & $40.16 \pm 2.17$ & $66.58 \pm 3.96^{\mathrm{a}}$ & $62.39 \pm 3.42^{\mathrm{a}}$ \\
72 & $44.90 \pm 2.47$ & $42.27 \pm 4.10$ & $47.58 \pm 3.66$ & $70.14 \pm 5.19^{\mathrm{a}}$ & $65.48 \pm 3.29^{\mathrm{a}}$ \\
EB1(h) & & & & & \\
24 & $35.54 \pm 2.17$ & $15.21 \pm 2.09$ & $37.43 \pm 4.30$ & $55.90 \pm 2.90^{\mathrm{a}}$ & $53.12 \pm 4.01^{\mathrm{a}}$ \\
48 & $37.91 \pm 3.34$ & $33.48 \pm 2.75$ & $37.41 \pm 4.21$ & $59.97 \pm 6.30^{\mathrm{a}}$ & $56.48 \pm 5.92^{\mathrm{a}}$ \\
72 & $44.38 \pm 6.21$ & $38.42 \pm 3.67$ & $42.84 \pm 3.09$ & $65.24 \pm 3.98^{\mathrm{a}}$ & $61.11 \pm 4.78^{\mathrm{a}}$ \\
\hline
\end{tabular}

${ }^{a} \mathrm{P}<0.05$; CCK8, cell counting kit-8; SD, standard deviation; siBCL2, BCL2 siRNA.

TableV. Rate of apoptosis by Annexin V analysis at $72 \mathrm{~h}$ after trancfection with BCL11A siRNA in combination with BCL2 siRNA in SUDHL6 and EB1 cells $(\%, \pm \mathrm{SD}, \mathrm{n}=3)$.

\begin{tabular}{lccccr}
\hline Cell lines & si585 & si2292 & siBCL2 & si585+siBCL2 & si2292+siBCL2 \\
\hline SUDHL6 & $32.00 \pm 3.15$ & $26.53 \pm 2.94$ & $25.27 \pm 1.49$ & $55.17 \pm 3.58^{\mathrm{a}}$ & $41.05 \pm 6.67^{\mathrm{a}}$ \\
EB1 & $29.43 \pm 2.38$ & $23.90 \pm 2.76$ & $18.29 \pm 2.39$ & $44.13 \pm 5.71^{\mathrm{a}}$ & $38.72 \pm 7.83^{\mathrm{a}}$
\end{tabular}

${ }^{\mathrm{a}} \mathrm{P}<0.05 ; \mathrm{SD}$, standard deviation; siBCL2, BCL2 siRNA.

the healthy control groups $(9,10)$. However, whether suppression of BCL11A was likely to induce apoptosis of cells has yet to be reported.

To clarify the role of BCL11A in B lymphoma cell proliferation and apoptosis, RNA interference was employed to inhibit the expression of BCL11A in two different human B-cell lymphoma-derived cell lines. Our results have shown that the expression levels of BCL11A mRNA and protein were decreased, following transfection of either SUDHL6 or EB1 cells with BCL11A siRNA. Two different siRNA targeting different regions (BCL11A-585, 2292) of the BCL11A sequence yielded congruent results. Viability of cells transfected with either BCL11A siRNA585 or 2292 showed a decrease in both SUDHL6 and EB1 cells. Moreover, findings of this study have also shown that the downregulation of BCL11A expression by siRNA is able to induce apoptosis in both SUDHL6 and EB1 cells. Taken together, the results suggest that BCL11A siRNA-mediated growth suppression of SUDHL6, EB1 cells occurs via the induction of apoptosis.

Our findings are in accordance with the known antiapoptotic role of BCL11B in T-cell malignancies (18-20). Recently, it has been shown that BCL11B is one of the necessary genes for T-cell differentiation and is associated with T-cell malignancies (21-23). BCL11B was 67\% identical to BCL11A at the nucleotide level and $61 \%$ identical overall at the protein level. Similar to BCL11A, BCL11B is the homologue of mouse CTIP-2 and is $86 \%$ identical at the protein level, which contains $6 \mathrm{C}_{2} \mathrm{H}_{2}$ zinc fingers and proline-rich and acidic regions with $95 \%$ identity in the zinc finger domains $(3,24)$. Therefore, we suggest that BCL111A and BCL11B has similar antiapoptotic action in malignant lymphocyte cells. However, the underlying molecular mechanisms by which BCL11A induces apoptosis remain to be elucidated. Taken together, our results have demonstrated that BCL11A may have an antiapoptotic role in malignant B lymphocyte cell lines. Our results therefore provide evidence for a major role of BCL11A in promoting the survival of malignant B cells.

BCL2 is known to be a prominent member of the BCL2 family which regulates the induction of apoptotic cell death by a wide variety of stimuli. Previous studies have shown that a reduction in BCL2 expression by siRNA is capable of inducing apoptosis in Raji cells (12). In the present study, as expected, BCL11A siRNA treatment in combination with BCL2 siRNA decreased cell proliferation and increased the number of apoptotic cells compared with BCL11A or BCL2 siRNA- transfected cells alone. Both these synergistic apoptotic effects and cell number reduction in the combination treatment are statistically significant. It has been shown that the blockage of multiple physiological events mediating malignancy and tumor cell survival, or blockage of a single critical path at multiple points to maximize inhibition of that path, is likely to result in greater-than-additive inhibition of tumor cell growth and viability (25). Similarly, in more recent studies, in vitro RNAi experiments conducted against hepatitis $\mathrm{C}$ virus using combinatorial treatment with two siRNAs elicited a significant increase in antiviral effects (26).

Our data indicate that the downregulation of BCL11A by siRNA induced apoptosis in SUDHL6, EB1 cells. BCL11A 
siRNA may therefore be useful for the treatment of tumors exhibiting an overexpression of BCL11A. Findings of the present study suggest that the selective blockade of BCL11A triggers apoptosis and suppresses the transduction of survival signals, leading to cancer cell growth inhibition and enhancing anti-tumor activity. Additionally, the combination of BCL11A and BCL2 siRNA produced a greater inhibitory effect on the cell proliferation of SUDHL6, EB1 cells compared to the two treatments given separately, indicating that BCL11A knockdown caused a marked synergistic enhancement of BCL2 siRNA-induced apoptosis. Our data therefore suggests that the downregulation of BCL11A expression is a relevant therapeutic strategy in B-cell lymphoma.

\section{Acknowledgements}

We would like to thank associate Professors Yubing Zhou and Lijian Yang (Medical College, Jinan University, Guangzhou, China) for kindly providing valuable technical support and advice. The study was supported by the Guangdong Province Key Foundation of Science and Technology Program (2009B0507000029), the Fundamental Research Funds for the Central Universities (no. 21610604), the National Natural Science Foundation of China (research project of cultivating project, no. 91129720) and grant from Overseas Chinese Affairs Office of the State Council Key Discipline Construction Fund (51205002).

\section{References}

1. Nakamura T, Yamazaki Y, Saiki Y,Moriyama M,Largaespada DA, Jenkins NA and Copeland NG: Evi9 encodes a novel zinc finger protein that physically interacts with BCL6, a known human B-cell proto-oncogene product. Mol Cell Biol 20: 3178-3186, 2000.

2. Liu P, Keller JR, Ortiz M, et al: Bcl11a is essential for normal lymphoid development. Nat Immunol 4: 525-532, 2003.

3. Satterwhite E, Sonoki T, Willis TG, et al: The BCL11 gene family: involvement of BCL11 A in lymphoid malignancies. Blood 98: 3413-3420, 2001.

4. Hystad ME, Myklebust JH, Bø TH, et al: Characterization of early stages of human B cell development by gene expression profiling. J Immunol 179: 3662-3671, 2007.

5. Martín-Subero JI, Gesk S, Harder L, et al: Recurrent involvement of the REL and BCL11A loci in classical Hodgkin lymphoma. Blood 99: 1474-1477, 2002.

6. WenigerMA,Pulford K, Gesk S, et al: Gains of the proto-oncogene BCL11A and nuclear accumulation of BCL11A(XL) protein are frequent in primary mediastinal B-cell lymphoma. Leukemia 20: 1880-1882, 2006.

7. Liu H, Ippolito GC, Wall JK, et al: Functional studies of BCL11A: characterization of the conserved BCL11 A-XL splice variant and its interaction with BCL6 in nuclear paraspeckles of germinal center B cells. Mol Cancer 5: 18, 2006.
8. Yin B, Delwel R, Valk PJ, Wallace MR, Loh ML, Shannon KM and Largaespada DA: A retroviral mutagenesis screen reveals strong cooperation between Bcl11a overexpression and loss of the Nf1 tumor suppressor gene. Blood 113: 1075-1085, 2009.

9. Gao Y, He D, Chen S, Yan X, Hu X and Li Y: Quantitative detection of BCL11A gene expression B cell malignancies by real-time RT-PCR. J Jinan Univ Med Ed 32: 147-150, 2011 (In Chinese).

10. Gao Y, He D, Chen S, Yan X, Hu X and Li Y: Expression of the B-cell lymphoma/leukemia 11A gene in malignant hematological cell lines through quantitative reverse transcription polymerase chain reaction. Clin Oncol Cancer Res 8: 242-246, 2011.

11. Kirkin V, Joos S and Zornig M: The role of Bcl-2 family members in tumorigenesis. Biochim Biophys Acta 1644: 229-249, 2004.

12. He D and Fang B: Small hairpin RNA targeting at Bcl-2 increases cytarabine-induced apoptosis in Raji cells. Acta Biochim Biophys Sin 41: 131-136, 2009.

13. Cioca DP, Aoki YJ and Kiyosawa K: RNA interference is a functional pathway with therapeutic potential in human myeloid leukemia cell lines. Cancer Gene Ther 10: 125-133, 2003.

14. Schmittgen TD and Livak KJ: Analyzing real-time PCR data by the comparative C(T) method. Nat Protoc 3: 1101-1108, 2008.

15. Livak KJ and Schmittgen TD: Analysis of relative gene expression data using real-time quantitative PCR and the 2(-Delta Delta C(T)) method. Methods 25: 402-408, 2001.

16. Xu J, Sankaran VG, Ni M, Menne TF, Puram RV, Kim W and Orkin SH: Transcriptional silencing of \{gamma\}-globin by BCL11A involves long-range interactions and cooperation with SOX6. Genes Dev 24: 783-798, 2010.

17. Sankaran VG, Menne TF, Xu J, et al: Human fetal hemoglobin expression is regulated by the developmental stage-specific repressor BCL11A. Science 322: 1839-1842, 2008.

18. Huang X, Chen S, Shen Q, et al: Down regulation of BCL11B expression inhibits proliferation and induces apoptosis in malignant $\mathrm{T}$ cells by BCL11B-935-siRNA. Hematology 16 : 236-242, 2011

19. Grabarczyk P, Przybylski GK, Depke M, et al: Inhibition of BCL11B expression leads to apoptosis of malignant but not normal mature T cells. Oncogene 26: 3797-3810, 2007.

20. Karanam NK, Grabarczyk P, Hammer E, et al: Proteome analysis reveals new mechanisms of Bcl11b-loss driven apoptosis. $\mathrm{J}$ Proteome Res 9: 3799-3811, 2010.

21. Li L, Leid M and Rothenberg EV: An early T cell lineage commitment checkpoint dependent on the transcription factor Bcl11b. Science 329: 89-93, 2010.

22. Huang X, Chen S, Shen Q, et al: Analysis of the expression pattern of the BCL11B gene and its relatives in patients with T-cell acute lymphoblastic leukemia. J Hematol Oncol 3: 44, 2010.

23. Karlsson A, Nordigården A, Jönsson JI and Söderkvist P: Bcl11b mutations identified in murine lymphomas increase the proliferation rate of hematopoietic progenitor cells. BMC Cancer 7: 195, 2007.

24. Avram D, Fields A, Top KP, Nevrivy DJ, Ishmael JE and Leid M: Isolation of a novel family of $\mathrm{C}(2) \mathrm{H}(2)$ zinc finger proteins implicated in transcriptional repression mediated by chicken ovalbumin upstream promoter transcription factor (COUP-TF) orphan nuclear receptors. J Biol Chem 275: 10315-10322, 2000.

25. Biroccio A, Leonetti C and Zupi G: The future of antisense therapy: combination with anticancer treatments. Oncogene 22: 6579-6588, 2003.

26. Xing XK, Li SJ, He JL and Chen Z: Inhibition of hepatitis C virus replication by single and dual small interfering RNA using an HCV-infected cell model. Biotechnol Lett 34: 295-301, 2012. 